$d^{2}$

\title{
Una perspectiva de la aplicación de la psicología de la salud en la rehabilitacion
}

* Cristina Camacho $F$.

\section{RESUMEN}

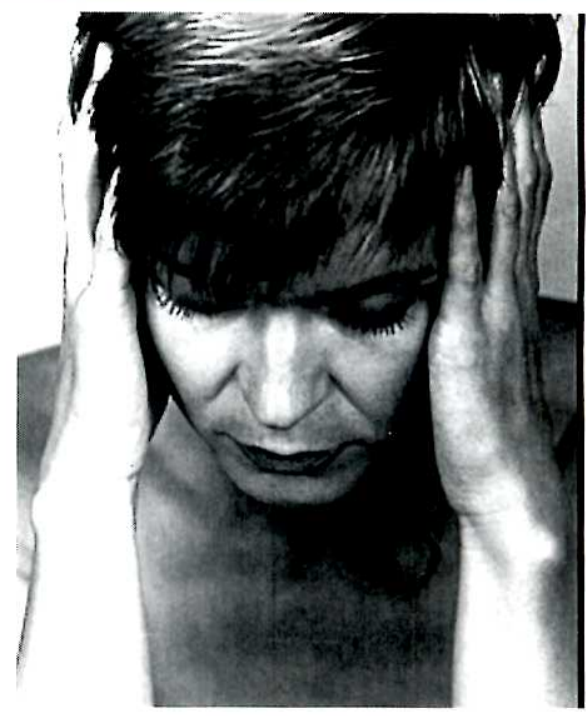

El artículo define la Psicología de la Salud y anota sus campos de interés, entre los cuales está la rehabilitación. Explica qué puede ofrecer, tanto al paciente como al equipo interdisciplinario en el proceso de rehabilitación y además anota ejemplos de los tipos de intervención psicológica con pacientes que presentan diferentes problemas de salud.

Palabras clave: Psicología en salud, conducta, rehabilitación.

\section{DEFINICIÓN DE TÉRMINOS}

El presente artículo define la psicología de la salud y busca establecer su relación con el campo de la rehabilitación física. La psicología es la ciencia que estudia el comportamiento humano y los estados psicológicos, es decir, los aspectos cognitivos, emocionales y conductuales. Para su estudio aplica el método científico; su objetivo básico es responder a la pregunta, "ipor qué las personas se comportan de la manera que lo hacen?". Para responder a ésta pregunta dentro de la psicología, existen varios campos como son: la psicología experimental, la social, la evolutiva, jurídica, clínica y de la salud entre otras.

Matarazzo (1884) anota que los aspectos psicológicos emocionales, cognitivos y comportamentales, son tan importantes como los aspectos físicos en el ser humano. El comportamiento en la forma de hábitos de salud, la búsqueda de cuidado médico y el cumplimiento de las recomendaciones médicas, para mencionar sólo algunas áreas, tienen un gran efecto sobre la salud. Los estados psicológicos también influyen directamente sobre esta, pues sentimientos y emociones positivos o negativos afectan el bienestar físico (Walltson y cols. 1983).

Justamente a partir de lo anterior es que surge la psicología de la salud surge como una especialización dentro de la psicología que trata con la salud física. A diferencia de la psicología clínica, que trata temas de salud mental, la psicología de la salud se centra en la salud física, en la relación dinámica entre comportamiento y estados psicológicos como lo aclara Bishop, (1994). La psicología de la salud también incluye el estudio del comportamiento y los estados psicológicos en la medida en que influyen y son influenciados por la salud física (Matarazzo, 1980).

De este modo, a la psicología de la salud le interesan varios campos: el primero, la promoción y mantenimiento de la salud; por esta razón presta mucha atención a los comportamientos de las personas, la manera como los hábitos - beber, hacer ejercicio, hacer dieta, fumar- afectan la salud.

En segundo lugar, se interesa en la prevención y tratamiento de las enfermedades, enseñando a las personas a manejar el estrés, a desarrollar buenos hábitos para la salud y a prevenir el desarrollo de enfermedades; ayuda a las personas en la rehabilitación a enfrentar su enfermedad, a recuperar su salud y funcionar lo mejor posible en los casos de problemas crónicos. En tercera instancia, se interesa por comprender los factores psicológicos que están

* Psicóloga clinica y de la salud, egresada de la Universidad Católica de Colombia y docente del Departamento de Humanidades, Institución Universitaria Escuela Colombiana de Rehabilitación. 
asociados con el desarrollo de la enfermedad y finalmente le interesa el desarrollo de los sistemas de atención en salud y la formulación de políticas de cuidado de la misma. (Bishop, 1994).

Desde su inicio, la psicología de la salud ha estado enmarcada dentro del modelo biopsicosocial, el cual considera la interacción de los factores biológicos, psicológicos y sociales en la prevención, tratamiento y rehabilitación de la enfermedad.

Es así, como en la prevención desarrolla programas para cambiar hábitos negativos de salud en las personas, motivándolas a adoptar hábitos positivos (estimulando el ejercicio regular y la dieta saludable en cambio del uso de sustancias tóxicas). En el tratamiento temprano de la enfermedad, define los comportamientos de la misma y cómo las personas interpretan los síntomas físicos y la enfermedad.

En la rehabilitación, entendida como el conjunto de procedimientos dirigidos a ayudar a la persona a alcanzar el más completo potencial físico, psicológico, social, vocacional y educacional, se hace compatible con su deficiencia a través de múltiples intervenciones dirigidas tanto a la causa como a los efectos secundarios de la lesión y la enfermedad; la psicología de la salud aplica los procedimientos de la psicología clínica, para tratar los problemas psicológicos asociados al desarrollo de la enfermedad.

\section{¿Qué ofrece la psicología de la salud a la rehabilitación?}

La psicología de la salud trabaja activamente en el desarrollo de nuevas formas para tratar con el dolor, las formas para manejo del estrés, y cómo la salud se ve afectada por las relaciones interpersonales. La tabla 1 a continuación muestra en síntesis qué ofrece frente a la enfermedad crónica.

1. Aprender a manejar el dolor, la incapacidad y otros síntomas

2. Tratar con el ambiente hospitalario y procedimientos de tratamientos especializados.

3. Desarrollar y mantener adecuadas relacionés con el personal de salud.

4. Preservar un balance emocional razonable.

5. Preservar auto-imagen satisfactoria y mantener un sentido de competencia.

6. Preservar relaciones familiares y sociales

7. Preparar para futuro incierto

TABLA 1 Tareas del Psicólogo de la Salud Frente a la Enfermedad Crónica)Tomado de Moos R.H. 1982
Diferentes habilidades deben ser desarrolladas por la persona durante la rehabilitación. Por ejemplo, como indica García (2000), la persona debe aprender a tratar con sus emociones de negación, culpa, miedo, entre otras, frente a la condición de salud y los procedimientos de rehabilitación. Se le enseña cómo las cogniciones y las sensaciones corporales (como el dolor) pueden provocar emociones que conducen a cambios en su conducta. Cuando estas conductas no son adaptativas a la situación, se entrena a la persona para que elabore interpretaciones cognitivas diferentes, que lleven a emociones y comportamientos más adaptativos. Aparte del tratamiento, la persona debe relacionarse adecuadamente con el equipo de profesionales de la salud, lo cual trae con frecuencia problemas de comunicación, asociados a la frustración frente a la enfermedad y al tratamiento por el temor a pedir información, aclarar dudas o establecer responsabilidades en el cumplimiento de objetivos; esto hace necesaria la intervención del psicólogo para entrenar en habilidades sociales básicas a la persona y en algunas ocasiones también al equipo de rehabilitación.

Igualmente, en muchas ocasiones es difícil para la persona mantenerse emocionalmente estable frente al proceso de rehabilitación, sobre todo, cuando éste conlleva maniobras complejas, incómodas y tratamientos prolongados. Se genera entonces estrés y a veces síntomas de depresión, frente a los cuales se pueden emplear técnicas cognitivo- conductuales que ayuden eficazmente a la persona. Relacionado con lo anterior, está la necesidad de preservar una autoimagen satisfactoria, y el sentido de competencia ("soy capaz de..."). Los cambios físicos que pueden presentarse, como ocurre por ejemplo en la artritis degenerativa, el lupus, las amputaciones, las quemaduras graves, etc. afectan negativamente la auto-imagen y estima de la persona. El apoyo psicológico se centra entonces, en ayudar a que redefina sus capacidades, haga una aceptación de su condición actual de salud, exprese sus necesidades y aprenda a mantener los máximos niveles de independencia. Lo anterior se logra a través de procedimientos de condicionamiento, auto-recompensa y apoyo social efectivo. De igual importancia, se considera brindar un entrenamiento a la familia y al grupo social y laboral, para que la persona complete su rehabilitación, reciba la aceptación y apoyo que merece. 


\section{Ejemplo de Casos}

A continuación se expondrán brevemente algunos ejemplos de casos en los cuales la psicología de la salud ayuda en la rehabilitación.

Primer caso: Control del estrés frente a un problema de hombro doloroso por traumatismo. El síndrome del estrés se define como una combinación de tres elementos: el ambiente, los pensamientos negativos y las respuestas físicas; el resultado es una emoción dolorosa (Dyer 1999). En este caso, el estímulo ambiental (mover el brazo para vestirse) produce una activación fisiológica (tensión muscular severa y dolor), seguido de una interpretación negativa (no puedo vestirme solo me siento inútil) y una emoción dolorosa (cólera). Se crea un círculo de retroalimentación negativo entre la mente y el cuerpo; la persona adopta posturas inadecuadas para reducir las sensaciones desagradables. Para romper el círculo, se le puede entrenar en relajación y visualización.

Segundo caso: Frente al paciente manipulador que presenta conductas infantiles y no quiere asumir la responsabilidad por su condición de salud; se requiere una modificación de comportamientos. Primero, establecer qué conductas se han estado reforzando y qué conductas deberían reforzarse, para que avance satisfactoriamente el proceso de rehabilitación. Segundo, para cambiar el comportamiento se debe dar un contracondicionamiento,(Gildenberg y cols., 1985) aplicar el condicionamiento operante, reforzar conductas deseables y retirar atención a las indeseables, de forma que la persona aprenda nuevamente cómo responder para recibir la atención deseada.

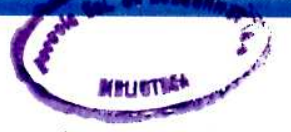

Tercer caso: Trastorno de ansiedad asociado a crisis de inflamación y dolor articular, acompañado de períodos de tiempo en que ha tenido que estar inmóvil en cama. En estos casos, además de tratar la enfermedad con medicamentos y terapia física, se hace necesario reducir la ansiedad asociada. Como bien anota en su folleto, el Instituto Nacional de Salud Mental (NIH) la terapia cognoscitiva- comportamental enseña al paciente a reaccionar en forma diferente ante las situaciones y sensaciones corporales que desencadenan la ansiedad; se aprende a cambiar pensamientos que anticipan la crisis y los síntomas de ansiedad y ayudan a enfrentar mejor las situaciones de miedo.

\section{Conclusiones}

La psicología de la salud, como especialidad dentro de la psicología que se centra en la interrelación dinámica que existe entre el comportamiento, los estados psicológicos y la salud física, contribuye en favor de la rehabilitación de personas con problemas motores. A través de la evaluación, el psicólogo de la salud diagnostica estados psicológicos de ansiedad o depresión, por ejemplo, y problemas de comportamiento asociados directa o indirectamente con el problema de salud física. Dentro del equipo multidisciplinario aporta los conocimientos teórico-prácticos para aplicar tratamientos cognitivos y comportamentales que ayuden al paciente que presenta deficiencias (secuelas patológicas) y discapacidades (restricciones en habilidades). Lo anterior, con el propósito de mejorar la calidad de vida del paciente y contribuir a su mayor nivel de independencia, estabilidad física y emocional general.

\section{BIBLIOGRAFIA}

Bishop, G.D. Exploring Heatlth Psychology. En Health Psychology: integrating mind and body. Allyn and Bacon (eds.) Boston (1984)

Dyer, $\boldsymbol{W} . \boldsymbol{W}$. Técnicas cognitivas para el tratamiento del estres. En www.ciudadfutura.com/psico/articulos

DeVellis, R.F. Social support and physical health. En Health Psychology,2 367-391. N.Y. (1983)

En www.angelfire.com/md2/rehabilitacion. (2001)

J.A., Miller, N.E. (eds). Behavioral Health: a handbook of health enhacement and disease prevention. N.Y. : Wiley (1984) Garcia, J.A. Relación cognición-sensaciones corporales dentro de la emoción: un condicionamiento clásico. (2000) En www.cop.es/coregrados

Gildenber, P.L., DeVaul, R.A.The chronic pain patient: evaluation and management. Ph.L. Gildenberg (ed.) Houston, Tex (1985) Matarazzo, J.D. Behavioral health and behavioral medicine: frontiers for a new health psychology. En American Psychologist, 35, 807-817 N.Y. (1980)

Matarazzo, J.D., Weiss, S.M., Herd, Moos, R.H. Coping with acute health crises. En T. Millon, C. Green, y R. Meagher (eds.) Handbook of clinical health psychology (p.135), N.Y.:Plenum (1982)

Trastornos de ansiedad. Folleto informativo de el "National Institute of Mental Health" (NIMH).En Wallston, B.S., Alagna, S.W., Fisiatría: Medicina Física y Rehabilitación.

www.montefiorecentral.com/WEBFINAL 